CASE REPORT

J.D. Barnwell

M. Castillo

\section{MR Imaging of Progressive Enhancement of a Bioceramic Orbital Prosthesis: An Indicator of Fibrovascular Invasion}

SUMMARY: The physical properties of bioceramics have made them ideal for a variety of prosthetic devices. Their porous structure allows fibrovascular tissue to invade the implant and secure it and provides a surface for muscular attachment. This process has been well-documented in animal studies; however, this case report describes the periodic imaging changes seen in a 67-year-old man following placement of a bioceramic orbital prosthesis.
$\mathbf{B}^{\mathrm{i}}$ ioceramics have been used in orthopedic and dental prosthetics for decades ${ }^{1}$; in 2000 , they were adapted to orbital prosthetics. Their aluminum oxide components form a cobblestone-appearing microcrystal structure that produces a lightweight, strong, easy-to-use, simple-tomanufacture, and highly porous implant. ${ }^{2}$ The pores of the implant allow fibrovascular invasion, which secures it within the orbital socket and provides a scaffold for muscular attachment. This process has been documented in animal studies that correlated changes in postcontrast T1 MR imaging with histologic samples demonstrating fibrovascular ingrowth. ${ }^{2-6}$ This report documents similar changes seen in a patient following right-sided enucleation and bioceramic prosthetic implantation.

\section{Case Report}

A 67-year-old man presented with chronic decreased visual acuity. A pigmented lesion, suspicious for melanoma versus choroidal nevus, was identified in the right eye. The lesion was followed with serial sonography for 9 years until its growth rate precluded the diagnosis of choroidal nevus. He then underwent right enucleation and bioceramic prosthetic implantation. Pathologic specimens confirmed the diagnosis of malignant melanoma. Lumbar punctures and imaging studies failed to demonstrate metastatic disease. Serial MR imaging studies with T1 spin-echo (TR, $400 \mathrm{~ms}$; TE, $15 \mathrm{~ms}$; 3-mm axial sections), T2 turbo spin-echo with fat saturation (TR, $5410 \mathrm{~ms}$; TE, 100 ms; echo train of 21; 3-mm axial sections), and postcontrast T1 spinecho with fat saturation (TR, $675 \mathrm{~ms}$; TE, $15 \mathrm{~ms}$; 3-mm axial sections following administration of gadobenate dimeglumines [MultiHance, $1 \mathrm{~mL} / 5 \mathrm{~kg}$; Bracco, Milan, Italy]) were performed on a 1.5 T magnet to evaluate the implant and assess disease recurrence. At 20 months after implantation, he is complication- and disease-free, and the prosthesis is well-incorporated clinically and on follow-up MR imaging (Fig 1).

\section{Discussion}

Fibrovascularization of orbital prosthetics secures them within the orbit and provides a surface for muscular attachment. This integral process has been examined in numerous animal models. These studies uniformly describe central T1

\section{Received September 26, 2009; accepted October 2.}

From the Department of Radiology, University of North Carolina at Chapel Hill School of Medicine, Chapel Hill, North Carolina.

Please address correspondence to James D. Barnwell, MD, 5625 Christie Ln, Durham, NC 27713; e-mail: james_barnwell@med.unc.edu

DOl 10.3174/ajnr.A1945 postcontrast enhancement, which coincides with histopathologic evidence of fibrovascularization. ${ }^{2-6}$

Jordan et $\mathrm{al}^{2}$ evaluated the bioceramic implant on 6 adult male New Zealand albino rabbits. The rabbits were divided into 3 groups. Each group had 1 rabbit with a bioceramic implant wrapped in polyglactin 910 (Vicryl; Ethicon, Cincinnati, Ohio) and 1 wrapped in the sclera of the specimen. The implants of the 3 groups were harvested at 4, 8, and 12 weeks, respectively. Each implant was imaged with a $1.5 \mathrm{~T}$ magnet by using 3-mm T1-weighted contrast-enhanced images before its removal. Following removal, the implants were weighed and bisected, and their internal and external surfaces were grossly evaluated. Histopathologic samples and scanning electron microscope images were obtained to assess fibrovascularization. All 6 specimens demonstrated central fibrovascularization as evidenced by central postcontrast enhancement on MR imaging.

Jordan et $\mathrm{al}^{3}$ performed a similar study on synthetic hydroxyapatite implants on 14 New Zealand white rabbits. The rabbits were also divided into 3 groups. Group $1(n=6)$ received a first-generation synthetic hydroxyapatite implant wrapped in Vicryl or sclera that was removed from 2 rabbits at 4,8 , and 12 weeks. Group $2(n=4)$ received a second-generation synthetic hydroxyapatite implant wrapped in Vicryl or sclera and removed at 4 and 12 weeks. Group $3(n=4)$ received a third-generation synthetic hydroxyapatite implant wrapped in Vicryl or sclera and removed at 4 and 12 weeks. Fibrovascularization was assessed via $1.5 \mathrm{~T}$ postcontrast $\mathrm{T} 1$ weighted MR imaging before removal and was confirmed with histopathologic specimens. Once again, central enhancement was seen by 12 weeks for the first generation implants, and 4 weeks for the second generation implants and third generation implants, which coincided with central fibrovascularization on histopathologic specimens. This model was repeated in 3 other studies with similar results. ${ }^{4-6}$

In our patient, MR imaging showed progressively lower T2 signal intensity in the prosthesis during the follow-up period. Additionally, postcontrast images demonstrated centripetal growth of enhancing tissues during the same period, compatible with fibrovascularization and incorporation of the prosthesis (Fig 1). Although this process was slower than that in the reported animal models, prosthetic incorporation timeframes in humans are not known. Additionally, although animal models demonstrated central enhancement, in our patient the enhancement started peripherally. Intuitively, this enhance- 


\section{Pulse Sequences}

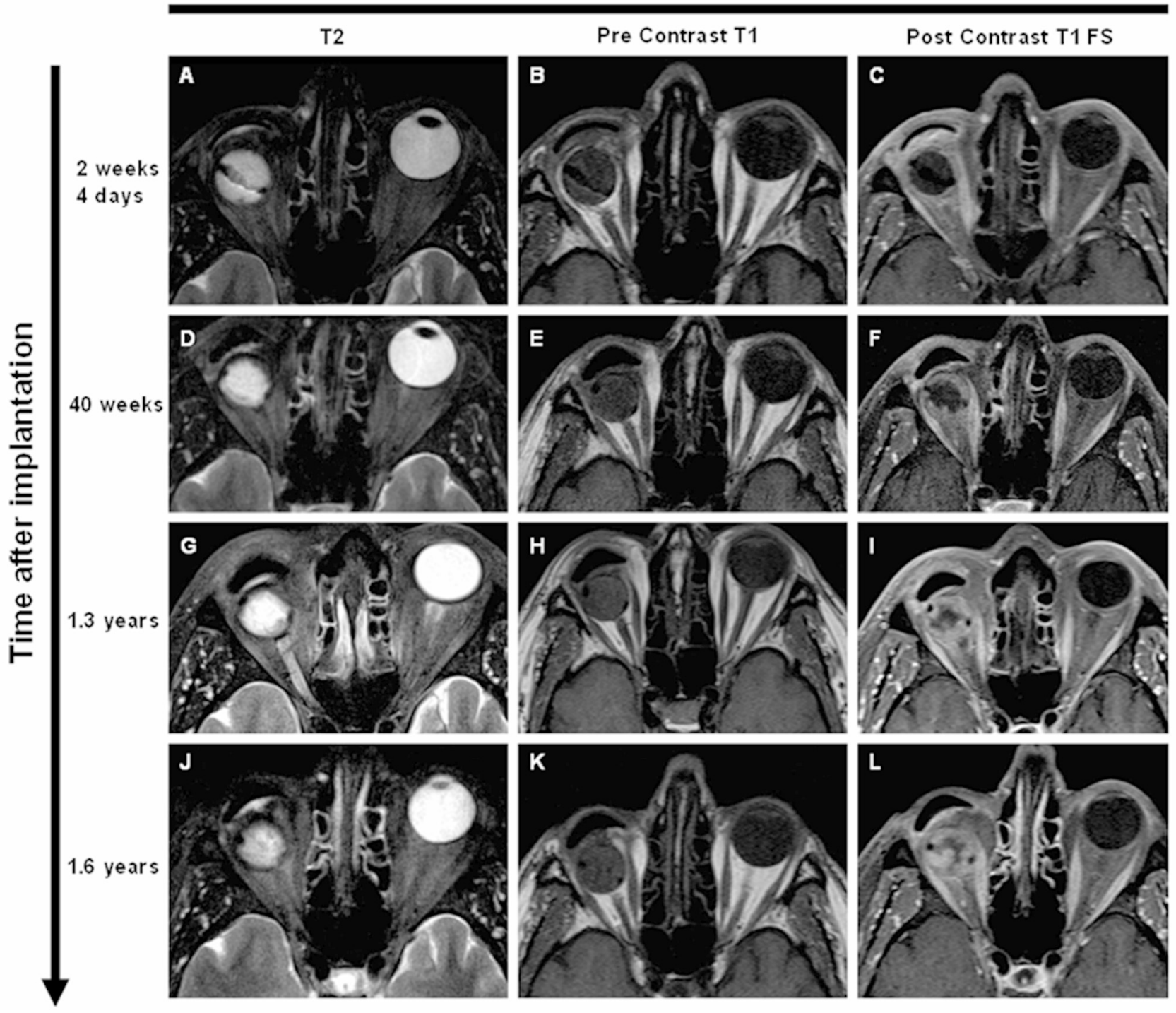

Fig 1. MR images of a patient with a right bioceramic orbital implant. The columns represent $\mathrm{T} 2$ turbo spin-echo with fat saturation $(A, D, G$, and $\mathcal{J})$, precontrast $\mathrm{T} 1$ turbo spin-echo ( $B$, $E, H$, and $K$, and postcontrast $T 1$ turbo spin-echo with fat saturation $(C, F, I$, and $L)$ images at different times. The rows represent the various time points when the patient underwent imaging following implant placement. There is a gradual decrease in T2 signal intensity and progression to central enhancement on contrast images due to in-growth of fibrovascular tissues and prosthetic incorporation.

ment makes sense because fibrovascular invasion should occur from the outside first. It is also possible that because the spacing of the studies performed in our patient differed significantly from those used in animal models, the patterns of enhancement may vary. Regardless of these differences, our limited experience with this patient seems to indicate that MR imaging with contrast may be used as a means of assessing the incorporation of a bioceramic orbital prosthesis. Progressive inward contrast enhancement correlated with good clinical prosthesis incorporation.

\section{References}

1. Christel P. Biocompatibility of surgical-grade dense polycrystalline alumina Clin Orthop 1992;282:10-18

2. Jordan D, Mawn L, Brownstein S, et al. The bioceramic orbital implant: a new generation of porous implants. Ophthal Plast Reconstr Surg 2000;16:347-55

3. Jordan DR, Munro S, Brownstein S, et al. A synthetic hydroxyapatite implant: the so-called counterfeit implant. Ophthal Plast Reconstr Surg 1998;14:244-49

4. Jordan DR, Pelletier C, Gilberg S, et al. A new variety of hydroxyapatite: the Chinese implant. Ophthal Plast Reconstr Surg 1999;15:420-24

5. Jordan DR, Hwang I, McEachren T, et al. Brazilian hydroxyapatite implant. Ophthal Plast Reconstr Surg 2000;16:363-69

6. Jordan DR, Hwang I, Brownstein S, et al. The Molteno M-sphere. Ophthal Plast Reconstr Surg 2002;16:356-62 